Review

\title{
Neurological Manifestations of the Celiac Disease in Children
}

\author{
Cristóbal Coronel-Rodríguez ${ }^{1,}{ }^{*}$, Alejandro Rodríguez-Martínez ${ }^{2}$
}

1. Centro de Salud "Amante Laffón", Sevilla, Spain; E-Mail: cristobalcoronel@gmail.com

2. Sección de Gastroenterología, Hepatología y Nutrición Pediátrica, Hospital Universitario Virgen del Rocío, Sevilla, Spain; E-Mail: armgastropediatria@gmail.com

* Correspondence: Cristóbal Coronel-Rodríguez; E-Mail: cristobalcoronel@gmail.com

Academic Editor: Luis Rodrigo

Special Issue: Gluten-Related Neurological Disorders

OBM Neurobiology

2020, volume 4, issue 3

doi:10.21926/obm.neurobiol.2003066
Received: May 05, 2020

Accepted: July 06, 2020

Published: July 17,2020

\begin{abstract}
Gluten-related disorders (GRDs) represent a spectrum of diverse clinical manifestations triggered by the ingestion of gluten in genetically susceptible individuals. The most common and widely recognized diseases within this spectrum are celiac disease and non-celiac gluten sensitivity. The clinical manifestations are intestinal, but extraintestinal manifestations may also appear, such as neurological or psychiatric.

This work will specifically review the neurological or psychiatric manifestations that are more frequently associated with GRD in children. Gluten ataxia, celiac disease, neuropathy, and celiac disease-epilepsy-brain calcifications syndrome are key entities that appear silently or oligosymptomatically on many occasions. However, they are more typical disorders in adult patients. The treatment with a gluten-free diet can usually alleviate the symptoms and, in some cases, even the neurological condition is cured.

Other conditions, such as headache, behavioral disorders, or generalized developmental disorders, are more typical in children. The pathophysiological basis of these conditions is not completely well-defined, although it is probably triggered by autoimmune mechanisms. The effective treatment approach is a gluten-free diet, although drugs such as antiepileptics, anti-migraine, immunomodulators, analgesics, or psychotropic drugs can control the clinical
\end{abstract}

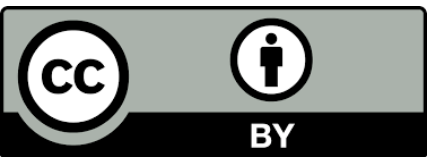

(C) 2020 by the author. This is an open access article distributed under the conditions of the Creative Commons by Attribution License, which permits unrestricted use, distribution, and reproduction in any medium or format, provided the original work is correctly cited. 
symptoms. The diagnosis of GRD should be made in the presence of neurological and psychiatric signs and symptoms, especially if they are of an unclear etiology. Also, the diagnosis should be made early, since their early correction can substantially improve the prognosis in some of these disorders.

\section{Keywords}

Celiac disease; non-celiac gluten sensitivity; child; ataxia; psychiatric disorders; neurological symptoms; calcifications

\section{Introduction}

Celiac disease (CD) is a systemic, chronic immune-mediated disorder elicited by gluten and related prolamines, which occurs in genetically susceptible individuals. Its clinical manifestations are mainly intestinal, but it may also be accompanied by extraintestinal manifestations. These extraintestinal manifestations are diverse and may be autoimmune or due to a deficit status. Most of these associated symptoms improve after switching to a gluten-free diet (GFD), although occasionally some of them also require specific treatment, either temporary or long-term [1].

$C D$ is one of the most common digestive disorders in children. Clinical features of $C D$ differ significantly depending on the age of presentation. Infantile CD usually appears during the first few years of life, when cereals containing gluten are introduced into the child's diet. Infantile CD generally adopts a "classic" clinical form characterized by typical malabsorption syndrome, followed by weight loss, profuse diarrhea, and signs of nutritional and vitamin deficiencies. However, CD in older children, adolescents, and young adults, tends to appear in a subacute or silent form, with fewer digestive symptoms and absence of malabsorption. It adopts a "nonclassic" form characterized by frequent extraintestinal manifestations and several associated diseases, which makes its diagnosis difficult [2].

Neurological disorders associated with gluten sensitivity, called "neurogluten" by some researchers, are neurological diseases related to permanent gluten intolerance, which appear in celiac patients or in people who have a non-celiac sensitivity to gluten (NCGS). These disorders can develop regardless of whether the person presents digestive symptoms or intestinal damage. Neurological presentations as the first signs of CD are rare in children, but as many as $36 \%$ of adult patients present with neurological changes [3, 4].

The clinical spectrum of neurological disorders is very broad, and the possibility that they may even be the first manifestation of the disease or appear as a complication of an already diagnosed and treated CD or NCGS makes it even more difficult to identify them. These disorders affect people of all ages; although they are usually more common in adults, they can also appear in children. At large, neurological manifestations of gluten-related diseases are approximately ten times more frequent in adults than those in the children. Neurological manifestations have been reported in approximately $6-10 \%$ of children with $C D$ according to the results of a systematic review and meta-analysis [5]. This difference is probably due to an early establishment of GFD and less cumulative exposure to gluten and its toxicity in children. These disorders have been known for a long time, and their presence has been widely documented. 


\section{Etiopathogenesis}

Several causes have been proposed for its etiopathogenesis in both adults and children, including nutritional, toxic, metabolic, and immunological causes. However, the data for or against each of them are scarce, and the origin of the neurological alterations is not always clearly known.

The malabsorption of certain components of the diet, such as tocopherol, cobalamin, or thiamine, has been reported. This type of deficiency is unusual, except in severe forms and with extensive involvement of the intestine. They may produce peripheral neuropathy (deficiencies of Vit B12 and thiamine) and demyelinating lesions of the central nervous system with an unclear mechanism. Its deficit could be a side effect of the CD malabsorption, and they could cause neurological deficit simply by its absence. The possible malabsorption of other factors or the excessive absorption of certain toxins has also been suggested as the origin of the neurological manifestations, including the accumulation of free radicals that produce oxidative stress. However, there is clear evidence to the contrary, such as the fact that these neurological manifestations can also appear in subjects with a histologically respected intestinal mucosa.

Gluten toxicity, and as a result, hyperHcy, can also be discussed in the pathogenesis of neurological disorders in CD. High Hcy levels have been reported in CD [6]. Hcy is a nonprotein sulfur-containing amino acid that is reversibly formed and secreted during methionine metabolism. It is hypothesized that hyperHcy might, by damaging the blood-brain barrier, cause neuronal exposure with neuro-irritative metabolites. Persisting damage to neuronal tissue can cause neurological disorders such as epilepsy and migraines [7].

The theory of the immunological origin of these neurological alterations has been put forward. The identification of tissue transglutaminase 2 (TG-2), the most relevant autoantigen in the pathogenesis of $C D$, has allowed further studies on the neurological manifestations of $C D$. Thus, in the absence of evidence to support a generalized disruption of the blood-brain barrier, the presence of autoantibodies in the cerebrospinal fluid of patients with CD would imply intrathecal production of immunoglobulins and supports an autoimmune origin of the neurological manifestations. In this regard, the discovery of a tissue transglutaminase at cerebral level (TG-6) and the presence of autoantibodies to TG-6 in patients with neurological symptoms suggest this mechanism. However, these results have limitations as they might have physiopathological significance, but the prevalence of these antibodies in asymptomatic individuals has not been studied, and it cannot be ruled out if they are epiphenomena [3].

\section{Clinical Manifestations}

Neurological clinical symptoms appear in a variable proportion of patients, but typically they are not very prevalent. The difficulty of diagnosis in the absence of intestinal disease and the broad clinical spectrum, suggests that this condition could be under-diagnosed, also in the pediatric patient. Neurological manifestations affect all stages of life and, although they tend to be more frequent in adults, they can also appear in children.

Contrary to the findings in adults, an increase in antigliadin, anti-transglutaminase, and antiendomysium antibodies has not been detected in children with unidentified neurological disorders in comparison to those of control (children without neurological manifestations). Therefore, in children with these disorders, it is questionable to initiate a differential diagnosis for $C D$, which should be reserved for more specific cases, such as the presence of lesions in the white substance 
or association with gastrointestinal symptoms $[8,9]$. On the other hand, the risk of developing neurological or psychiatric disorders once treated with a GFD is low in children, approximately $2.6 \%$ against $26 \%$ in adults [10]. This difference may be due to the early elimination of gluten and the protective effect of the diet on children who are less exposed to gluten and active $C D$, compared to that of adults with delayed diagnosis.

Next, we describe the most frequently associated neurological manifestations related to $C D$ in pediatrics:

\subsection{Epilepsy}

Several studies indicate an association between the presence of $C D$ and various forms of epilepsy, and it is one of the earliest known neurological manifestations of the disease [11]. This association seems to happen more frequently in children than in adults. Epilepsy is a common chronic neurological disease with an estimated prevalence ranging from $0.6 \%$ to $1.7 \%$. In different studies, the overall prevalence of epilepsy in celiac patients was $0.79-1 \%$. However, among those with paucisymptomatic $C D$, in which the diagnosis is made earlier, the prevalence was $1.6 \%$, and among asymptomatic CD patients it was 3.5\% [12]. These data suggest that, if the disease is diagnosed late, with a longer time of exposure to gluten, there is a greater risk of developing epilepsy.

Seizures are usually partial, either simple or complex, with or without secondary generalization. Usually, they affect the occipital lobe, and seizures originating in the temporal lobe are less common. Even though primarily generalized seizures and other types of epileptic syndromes may occur, they are not common [13, 14]. Occipital lobe seizures may be due to calcifications in the region and may respond to GFD. Calcifications are usually bilateral, symmetrical, and they are located in the cortical and subcortical regions. Despite being a very characteristic association, it is very rare. If we consider $C D$ diagnosed patients, the proportion of them with epilepsy and occipital calcifications, as seen in neuroimaging, is very low, which indicates it may be a different syndrome. Some authors consider this to be a specific and different syndrome from $C D$, and have even proposed the name "CEC syndrome (CD-epilepsy-cerebral calcification)" or "Gobbi syndrome." Other authors believe that the association of epilepsy and occipital calcifications without an intestinal lesion is a silent or potential form of a $C D$ on the basis that both isolated epilepsycerebral calcifications and CD are associated with HLA-DQ2 and DQ8. This association was first described in 1992, and since then, fewer than 200 patient cases have been documented worldwide, including pediatric patients $[15,16]$.

With respect to other epileptic syndromes, it is not clear whether they are more frequent in celiac patients or if there is only an accidental association between CD and epileptic seizures in children where gluten toxicity may contribute to a higher number of seizures. The response to GFD varies according to the studies, from significant clinical improvement to partial improvement, in patients where epilepsy has earlier been established. Improvement usually occurs with a decrease in frequency and intensity of seizures, although these often do not completely disappear. The effect of GFD on seizure control seems to be inversely related to the length of epilepsy and patients' age $[17,18]$. 


\subsection{Ataxia}

In adult patients, gluten ataxia accounts for $40 \%$ of ataxias of unknown origin and $15 \%$ of all ataxias $[19,20]$. It is one of the neurological disorders most frequently associated with CD, and though it can appear in people of all ages, including children [21], it is generally of late-onset. In a meta-analysis conducted by Lionetti et al. [22], the presence of gluten-based cerebellar ataxia was documented in two large studies and reported in $2.7 \%$ and $5.4 \%$ of patients, respectively. However, no correlation between CD and ataxia was found in two other studies of the analysis.

The mean age of onset is usually around 50 years, and it is three times more frequent in males. The clinical profile involves cerebellar participation of the posterior cordons of both the spine and peripheral nerves, causing balance issues, gait, instability, trembling limbs, and nystagmus, which prevent patients from leading a normal life. Clinically, no difference has been found with ataxia of other origins.

The causes of cerebellar involvement have not yet been well established. At present, it seems most likely that its etiology is of immune origin. Supporting this, studies have shown that patients with gluten ataxia have antibodies against the cerebellum Purkinje cells that affect, in a more subtle way, the neurons of the cerebral cortex and deep nucleus of the cerebellum. This is consistent with the loss of Purkinje cells that have been demonstrated by necropsies [23]. It has also been shown that both antigliadin antibodies and serum from most patients with gluten ataxia react against Purkinje cells. Cross-reactivity of these antibodies would indicate that gliadin proteins and Purkinje cells share common epitopes. These common epitopes between gliadin proteins and enterocytes have also been demonstrated, as well as several genes with partial homology to gliadin [24]. However, the presence of elevated antigliadin antibodies can also be seen in diseases such as Huntington's chorea or genetically determined spinocerebellar ataxias. These findings question the etiopathogenic value of antibodies in the genesis of ataxia. Other antibodies, such as anti-gangliosides, which are implicated in other neurological manifestations of $C D$, have not been associated with either ataxia or gluten hypersensitivity [25]. More recently, the role of transglutaminase (TG) in the genesis of gluten ataxia has been studied. Transglutaminase is a calcium-dependent enzyme found in blood vessels and the brain. It is involved in tissue restoration, acute phase response, and maintenance of vascular permeability. The findings in necropsy material from patients with gluten ataxia have demonstrated loss of Purkinje cells, perivascular lymphocyte infiltration, and deposition of anti-TG-6 IgA [26]. Some studies replicate gluten ataxia in animal models by injecting serum or isolated antitranglutaminase antibodies from gluten ataxia patients or CD patients [27].

It has been accepted that in patients with ataxia of unknown origin, gluten-related diseases should be ruled out. Currently, it has been recommended that patients with cerebellar ataxia should be typed for HLA and assessed for the presence of anti-gliadin (IgA and IgG), antiendomysium, and anti-TG (preferably anti-TG-6) antibodies [20]. Only $10 \%$ of patients with ataxia report gastrointestinal symptoms; however, one-third have evidence of celiac enteropathy on biopsy $[28,29]$.

If no other cause of cerebellar ataxia can be determined and there is positivity for any of these antibodies, a GFD is strictly recommended. If antibody titers decrease or there is either noticeable improvement or stabilization of ataxia, a diagnosis of gluten ataxia should be acceptable [30]. The improvement in ataxic symptoms may be variable and depends on the duration of the disease, as 
the loss of Purkinje cells and detectable atrophy are not reversible. The response to GFD is very good when it is established early (within the first six months). When it is done later, there is usually no complete improvement. In the case of pediatric patients, the presence of neurological sequelae after the beginning of GFD is exceptional.

\subsection{Peripheral Nervous System Involvement}

Very few studies have addressed the involvement of the peripheral nervous system in the prevalence of $C D$ in children, and its possible association with gluten-related diseases. Cakir et al. showed in their series that up to $7.4 \%$ of children with $C D$, whether they followed a GFD adequately or not, had a peripheral axonal polyneuropathy with mixed sensory-motor or pure sensory patterns [31]. In contrast, Ruggieri et al. reported a single case in a series of 835 children with $\mathrm{CD}(0.1 \%)$, of a girl with a motor axonal polyneuropathy who experienced relapses when she accidentally consumed gluten in her diet and whose condition rapidly remitted when strict GFD was re-established [14]. A series of adults similarly related to both conditions, with two ends being evident: one in which neuropathy is described in almost half of the patients, and another in which no such association has been found $[32,33]$.

The involvement of peripheral nervous system usually manifests as polyneuropathy, generally axonal and predominantly sensory, although some cases have also been reported as mononeuritis multiplex, pure motor neuropathy, Guillain-Barré syndrome, and autonomic neuropathy.

From an etiopathogenic point of view, and as in gluten ataxia, an immune basis is recognized, and a relationship with anti-TG-6 autoantibodies has been described [34]. In other studies, different forms of polyneuropathy with $C D$ have been associated with the presence of specific IgG autoantibodies to GM1, GM2, GD1a, and GD1b [35]. The effect of GFD on other neurological manifestations is not clear, describing cases in children, where there has been a significant clinical improvement in some, as well as others where this is not observed [36].

\subsection{Headache}

Although headache is a non-specific symptom, a higher proportion of CD has been diagnosed in migraine patients than in healthy patients, and a higher proportion of headache has been demonstrated among CD patients than in the general population [37]. In a series of children with $\mathrm{CD}$, Lionetti et al. found a higher prevalence of headache at the time of diagnosis compared to healthy individuals $(24.8 \%$ vs. $8 \% ; p=0.001)$ [38]. These data are consistent with those reported by Zelnik et al. in the previous studies [4]. In particular, in a meta-analysis of the available evidence, the RR of patients with $C D$ to develop headache was 3.2 (95\% Cl 2.2-4.7), the OR was 4.0 (95\% Cl 2. 6-6.1; $\mathrm{P}<0.001, \mathrm{I} 2<27.6 \%)$, and the RD of $0.08(95 \% \mathrm{Cl} 0.06-0.11 ; \mathrm{P}<0.001, \mathrm{I} 2=$ $99.7 \%)$, indicating that children with CD may be at a higher risk of suffering from headaches [38].

If these relationships were confirmed, a headache would become a neurological disorder most commonly associated with gluten-related diseases. Again, the etiology is unclear and does not appear to be related to micronutrient deficiencies. The physiopathology of this association is more likely linked to immunological phenomena. Hadjivassiliou et al. described hyper-intensities in the white matter of patients with migraines and CD, which improved with diet [39]. Headache and migraine generally show a significant improvement after initiating a strict GFD. The prevalence of stress headaches also seems to be higher in patients with CD [40]. 


\subsection{Autism}

Autism is a neurodevelopmental disorder with onset in childhood. Its etiology is unclear, but it appears to be caused by the interaction of inherited genetic susceptibility, epigenetic processes, and environmental factors acting during embryogenesis. It is characterized by altered development of social communication, behavioral disorders, and repetitive and restricted behaviors and interests. It has a chronic evolution, with different degrees of involvement, functional adaptation, and functioning in the area of language and intellectual development, depending on the case and evolutionary movement [41].

The association between autism and $C D$ is controversial, with no studies confirming this association. The age of onset, symptoms recognition, the improvement with GFD, and the worsening of behavior when gluten is reestablished have generated great confusion. In a study performed by Pavone et al. with 120 CD patients, none of them were diagnosed with autism [42]. More recently, Ludvigsson et al. found no association between CD or intestinal inflammation and autism, although they did observe a markedly increased risk of autism in those with positive serological tests but normal mucus [43].

On the other hand, Lau et al. found that antigliadin antibodies were higher in children with autism and gastrointestinal symptoms than those in the control group, those with gliadin antideaminating antibodies and anti-TG-2 did not show such differences. They also found no difference in HLA-DQ2 and/or DQ8 [44]. Therefore, there is no sufficient evidence to support an association between autism and CD, so an association between GFD and autism is not justified. However, a thorough examination and clinical studies should be carried out in search of specific antibodies or enteropathy, since, in the event of a positive diagnosis, GFD will be essential and will probably improve some of the symptoms.

\subsection{Lesions in the White Matter}

Kieslich et al. used neuroimaging techniques to study children with CD who underwent GFD. Uni- and bilateral focal lesions in the brain white matter were detected in $20 \%$ of the participants. The existence of these lesions was not correlated with the patient's dietary compliance, the presence of neurological symptoms, or with the presence of electroencephalographic recording alterations; however, the average exposure to gluten was longer in participants who presented lesions than in those who did not [9].

Ruggieri et al. also studied these lesions and registered three children with CD and associated neurological symptoms; two children had associated psychiatric disorders and poor diet compliance, and one had headache [14]. Subsequently, isolated cases with these same lesions have been reported, with variable response to GFD [7]. The origin of these lesions is unknown. Some authors suggest that the origin may be ischemic, as a result of an immunologically mediated process of vasculitis, although it has also been proposed that the origin may be secondary demyelination.

In any case, these focal lesions of the brain white matter also appear to be an extraintestinal manifestation of the $C D$ because these phenomena have also been described in other immunemediated chronic gastrointestinal diseases [45]. 


\subsection{Psychiatric Disorders}

Psychiatric disorders associated with CD have been known for years and appear in up to onethird of adult patients [46]. The most frequently reported symptoms in children are apathy, anxiety, and irritability. Whereas, major depression, dysthymia, adjustment or panic disorders, recurrent depressive episodes, schizophrenia, eating disorders (anorexia and bulimia nervosa), altered sexual habits, and feelings of sadness, anger, or fear are observed in adults [5].

According to a recent cohort study, children with untreated $C D$ are more likely to have behavioral disturbances than their healthy peers $(\mathrm{H} \mathrm{1.7,95 \%} \mathrm{Cl} 1.4-2.1)$. Also, those children are more likely to suffer from depression $(\mathrm{H}=1.8,95 \% \mathrm{Cl} 1.6-2.2)$ and more likely to present mood disorders (H 1.2, 95\% Cl 1.0-1.4) [47].

By contrast, most cross-sectional studies have shown that the prevalence of depression or the severity of depressive symptoms does not differ in children with CD compared to their controls [48]. In a cross-sectional study by Pynnonen et al., no difference was found between the prevalence of depression in pediatric patients with $\mathrm{CD}$ and their controls, but it was found in lifetime prevalence ( $31 \%$ vs. $7 \%$; $O R=6.06,95 \% \mathrm{Cl} 1.18-31.23$ ) [49]. Similarly, regarding anxiety disorders, although some cross-sectional studies have shown no difference between patients with $\mathrm{CD}$ and healthy patients, cohort studies show a higher probability of occurrence of these disorders (H 1.2, 95\% Cl 1.0 to $1.4, \mathrm{p}<0.05)$ [47].

The association between $C D$ and eating disorders in children has also been described $(H$ 1.4, $95 \% \mathrm{Cl} 1.1$ to 1.8 ), as well as a slight but significant probability of presenting an attention deficit hyperactivity disorder [47]. In children, no association has been found between CD and the occurrence of bipolar disorder or psychotic disorders [50].

The origin of these alterations in the psychiatric sphere of $C D$ has been related to a deficiency of certain vitamins and immunological disorders. Tryptophan and other monoamines are lacking in serum and cerebrospinal fluid (CSF) of patients with untreated CD. Cases have been described in which symptoms have improved with GFD; others have improved with vitamin supplements without GFD and another group in which GFD improved anxiety but not depression. These data suggest that psychiatric symptoms associated with CD may have a heterogeneous origin. It seems reasonable to consider that any child with psychiatric symptoms without clear origin, or that do not improve with treatment, may have a silent or potential form of $C D$, and it is advised to investigate its presence.

\subsection{Others}

Muscle involvement associated with $C D$ has also been described in children. Delayed acquisition of psychomotor milestones and hypotonia appear to be the only neurological symptoms that appear more in children than in adults, and it has been suggested that they may be related to atrophy and deficit in vitamins, ions, and micronutrients. Some of these disorders, such as hypotonia, resolve after years of GFD.

Another symptom strongly associated with $C D$ is asthenia, especially in adolescents, which may also cause depression. This symptom often goes unnoticed on many occasions, leading to a delay in diagnosis. Besides, it appears to respond well to GFD [51]. 


\section{Diagnosis}

\subsection{Immunological and Histological Study}

It seems reasonable to perform an immunological study on a large number of patients, taking into account the fact that neurological symptoms of unclear origin, therefore, CD or other glutenrelated disorders, may be under-diagnosed.

Currently, the determination of anti-TG-2 IgA antibodies along with the quantification of serum IgA constitutes the reference test for serological diagnosis. The recently published New European Society of Pediatric Gastroenterology, Hepatology, and Nutrition (ESPGHAN) criteria recommend against deamidated gliadin peptide antibodies (DGP-IgG/IgA) for initial testing. Only if total $\lg A$ is low/undetectable, an IgG based test is indicated. Patients with positive results should be referred to a pediatric gastroenterologist/specialist. If TGA-IgA is $\geq 10$ times higher than the upper limit of normal $(10 \times U L N)$ and if the family agrees, the no-biopsy diagnosis may be applied, provided endomysial antibodies (EMA-IgA) will test positive in a second blood sample. HLA DQ2-/DQ8 determination and symptoms are not obligatory criteria. In children with positive TGA-IgA $<10 \times U L N$, at least four biopsies from the distal duodenum and at least one from the bulb should be taken. Discordant results may require re-evaluation. Patients with no/mild histological changes (Marsh 0/I) but confirmed autoimmunity (TGA-IgA/EMA-IgA+) should be followed closely. In cases with type 1 diabetes mellitus or cases with IgA deficit, the possibility of diagnosis without intestinal biopsy is not considered [52].

It has been accepted that in patients with ataxia of unknown origin, the CD should be ignored. The diagnosis of "gluten ataxia" is shown by elevated blood autoantibody levels associated with injury to the intestinal mucosa, such as anti-endomysium, antigliadin, or anti-TG-2, even in the absence of intestinal disease. Other authors suggest that it is necessary to prove an improvement or stabilization of the clinical symptoms after initiating GFD to establish this diagnosis.

\subsection{Brain Imaging Tests}

In some cases, neuroimaging tests such as MRI show non-specific generalized atrophy, which affects the cerebellum more severely in cases of ataxia. They also show occipital calcifications, which are frequent in cases of CEC syndrome. These are usually corticosubcortical and are not enhanced after contrast application. Alterations in the white matter, with preservation of the hippocampus, have also been shown to be probably related to ischemic lesions as a result of vasculitis or post-inflammatory demyelination. Imaging tests have been used for patient follow-up and to monitor response to treatment.

\subsection{Neurophysiological Testing}

Focal or generalized abnormalities can be recorded on the electroencephalogram, and it is used to follow up patients with epilepsy and to monitor treatment. The use of stereotactic deep electrodes may be useful in the study of patients who are candidates for surgery of refractory epilepsy associated with occipital calcifications. Electromyograms may target polyneuropathy, which, as already mentioned, is typically axonal and mildly sensory-motor. However, it should be 
taken into consideration that the electrophysiological study in patients with a gluten-related disorder may be normal or almost normal in patients even with clinical polyneuropathy.

\subsection{Study of the Cerebrospinal Fluid}

The CSF study is usually very non-specific. The role of antigliadin and antiTG- 6 autoantibodies in the CSF remains unclear, as they have been detected in the population with $C D$, and their prevalence in the healthy population has not been studied.

\section{Treatment}

\subsection{Strict Gluten-Free Diet}

The different neurological manifestations associated with GFD vary between individuals. GFD shows poor results in epilepsy not associated with occipital calcifications or in cerebellar ataxia of long evolution. Other symptoms, such as headache, asthenia, depression, seem to respond better to GFD.

Irreversible neurological manifestations are observed in patients in whom the diagnosis is made late, and the start of GFD is delayed. For this reason, it is exceptional to see pediatric patients who do not respond to GFD.

Since CD can also be associated with vitamin deficiency, it may be advisable to treat with vitamin supplements. It is also recommended to determine analytical parameters such as ferritin, vitamin B12, folic acid, calcemia, and vitamin D. In case of deficiency or insufficiency, supplementation is recommended.

\subsection{Immunomodulators}

Some patients show a good response to cerebellar ataxia treatment with intravenous immunoglobulins [53]. Medications such as corticosteroids, immunosuppressants (cyclosporine, azathioprine), or biological drugs (infliximab) have shown good results for treating CD of other extraintestinal locations, although the effects they might have on the nervous system involvement are not yet known.

\subsection{Surgery}

Surgical removal of calcifications is indicated as a treatment for untreatable comitological crises of occipital or temporal lobe origin, for which the presence of laterality is required.

\subsection{Symptomatic Treatment}

Depending on the patient's specific symptom, combinations of antiepileptic, anti-migraine, antidepressant, antipsychotic, or analgesic medications are prescribed to improve the patient's baseline conditions. 


\section{Conclusions}

The spectrum of neurological disorders in pediatric patients with CD is broader now than it was before but much narrower than that described in the adult population. Neurological disorders range from previously known entities such as epilepsy or cerebellar ataxia to more subtle disorders such as headache, behavioral disorder or attention deficit, and hyperactivity disorder.

Therefore, the diagnosis of CD should be considered in the presence of neurological disorders, especially those of an unclear etiology, and should be made early, given the possible treatment of the disease. Emphasis should be placed on including differential diagnosis of multiple disorders, to familiarize specialists with the neurological manifestations in this entity.

\section{Author Contributions}

C.C. conceived the original idea. A.R. took the lead in writing the manuscript. Both authors provided critical feedback and helped shape the review, analysis and manuscript.

\section{Competing Interests}

The authors have declared that no competing interests exist.

\section{References}

1. Rodrigo Sae L, Lauret-Braña M, Pérez-Martínez, I. Manifestaciones extra-intestinales y enfermedades asociadas. OmniaScience Monographs. 2012.

2. Rodrigo Sáez L, Fuentes Álvarez D, Pérez Martínez I, Álvarez Mieres N, Niño García P, Francisco García RD, et al. Diferencias entre la enfermedad celiaca infantil y del adulto. Rev Esp Enfermedades Dig. 2011; 103: 238-243. doi:10.4321/S1130-01082011000500003

3. Bermejo VP, Burgos GA. Complicaciones neurológicas de la enfermedad celíaca. Med Clínica. 2006; 127: 500-507. doi:10.1157/13093268

4. Zelnik N, Pacht A, Obeid R, Lerner A. Range of neurologic disorders in patients with celiac disease. Pediatrics. 2004; 113: 1672-1676. doi:10.1542/peds.113.6.1672

5. Trovato CM, Raucci U, Valitutti F, Montuori M, Villa MP, Cucchiara S, et al. Neuropsychiatric manifestations in celiac disease. Epilepsy Behav. 2019; 99: 106393. doi:10.1016/j.yebeh.2019.06.036

6. Saibeni S, Lecchi A, Meucci G, Cattaneo M, Tagliabue L, Rondonotti E, et al. Prevalence of hyperhomocysteinemia in adult gluten-sensitive enteropathy at diagnosis: Role of B12, folate, and genetics. Clin Gastroenterol Hepatol. 2005; 3: 574-580. doi:10.1016/s15423565(05)00022-4

7. Sel ÇG, Aksoy E, Aksoy A, Yüksel D, Özbay F. Neurological manifestations of atypical celiac disease in childhood. Acta Neurol Belg. 2017; 117: 719-727. doi:10.1007/s13760-017-0781-z

8. Lahat E, Broide E, Leshem M, Evans S, Scapa E. Prevalence of celiac antibodies in children with neurologic disorders. Pediatr Neurol. 2000; 22: 393-396. doi:10.1016/s0887-8994(00)00129-6

9. Kieslich M, Errázuriz G, Posselt HG, Moeller-Hartmann W, Zanella F, Boehles H. Brain whitematter lesions in celiac disease: a prospective study of 75 diet-treated patients. Pediatrics. 2001; 108: e21. doi:10.1542/peds.108.2.e21 
10. Ertürk E, Wouters S, Imeraj L, Lampo A. Association of ADHD and celiac disease: What is the evidence? A systematic review of the literature. J Atten Disord. 2016. doi:10.1177/1087054715611493

11. Chapman RW, Laidlow JM, Colin-Jones D, Eade OE, Smith CL. Increased prevalence of epilepsy in coeliac disease. Br Med J. 1978; 2: 250-251. doi:10.1136/bmj.2.6132.250

12. Tengah DSNAP, Holmes GKT, Wills AJ. The prevalence of epilepsy in patients with celiac disease. Epilepsia. 2004; 45: 1291-1293. doi:10.1111/j.0013-9580.2004.54104.x

13. Mavroudi A, Antigoni M, Xinias I, Karatza E, Maria F, Panteliadis C, et al. Increased prevalence of silent celiac disease among Greek epileptic children. Pediatr Neurol. 2007; 36: 165-169. doi:10.1016/j.pediatrneurol.2006.11.011

14. Ruggieri M, Incorpora G, Polizzi A, Parano E, Spina M, Pavone P. Low prevalence of neurologic and psychiatric manifestations in children with gluten sensitivity. J Pediatr. 2008; 152: 244249. doi:10.1016/j.jpeds.2007.06.042

15. Johnson AM, Dale RC, Wienholt L, Hadjivassiliou M, Aeschlimann D, Lawson JA. Coeliac disease, epilepsy, and cerebral calcifications: association with TG6 autoantibodies. Dev Med Child Neurol. 2013; 55: 90-93. doi:10.1111/j.1469-8749.2012.04369.x

16. Arroyo HA, De Rosa S, Ruggieri V, De Dávila MT, Fejerman N. Epilepsy, occipital calcifications, and oligosymptomatic celiac disease in childhood. J Child Neurol. 2002; 17: 800-806. doi:10.1177/08830738020170110801

17. Pratesi R, Modelli IC, Martins RC, Almeida PL, Gandolfi L. Celiac disease and epilepsy: favorable outcome in a child with difficult to control seizures. Acta Neurol Scand. 2003; 108: 290-293. doi:10.1034/j.1600-0404.2003. 00082.x

18. Mavroudi A, Karatza E, Papastavrou T, Panteliadis C, Spiroglou K. Successful treatment of epilepsy and celiac disease with a gluten-free diet. Pediatr Neurol. 2005; 33: 292-295. doi:10.1016/j.pediatrneurol.2005.05.010

19. Hadjivassiliou M, Sanders DD, Aeschlimann DP. Gluten-related disorders: gluten ataxia. Dig Dis. 2015; 33: 264-268. doi:10.1159/000369509

20. Hadjivassiliou M, Martindale J, Shanmugarajah P, Grünewald RA, Sarrigiannis PG, Beauchamp $\mathrm{N}$, et al. Causes of progressive cerebellar ataxia: Prospective evaluation of 1500 patients. J Neurol Neurosurg Psychiatry. 2017; 88: 301-309. doi:10.1136/jnnp-2016-314863

21. Gordon N. Cerebellar ataxia and gluten sensitivity: A rare but possible cause of ataxia, even in childhood. Dev Med Child Neurol. 2000; 42: 283-286. doi:10.1017/s0012162200000499

22. Lionetti $E$, Francavilla $R$, Pavone $P$, Pavone $L$, Francavilla $T$, Pulvirenti $A$, et al. The neurology of coeliac disease in childhood: What is the evidence? A systematic review and meta-analysis. Dev Med Child Neurol. 2010; 52: 700-707. doi:10.1111/j.1469-8749.2010.03647.x

23. Hadjivassiliou M, Boscolo $S$, Davies-Jones GA, Grünewald RA, Not TA, Sanders DS, et al. The humoral response in the pathogenesis of gluten ataxia. Neurology. 2002; 58: 1221-1226. doi:10.1212/wnl.58.8.1221

24. Kumar R, Lumsden A, Ciclitira PJ, Ellis HJ, Laurie GW. Human genome search in celiac disease using gliadin cDNA as probe. J Mol Biol. 2000; 300: 1155-1167. doi:10.1006/jmbi.2000.3927

25. Shill HA, Alaedini A, Bushara KO, Latov N, Hallett M. Anti-ganglioside antibodies in idiopathic and hereditary cerebellar degeneration. Neurology. 2003; 60: 1672-1673. doi:10.1212/01.wnl.0000060185.30001.46 
26. Hadjivassiliou M, Aeschlimann P, Sanders DS, Mäki M, Kaukinen K, Grünewald RA, et al. Transglutaminase 6 antibodies in the diagnosis of gluten ataxia. Neurology. 2013; 80: 17401745. doi:10.1212/WNL.0b013e3182919070

27. Boscolo S, Lorenzon A, Sblattero D, Florian F, Stebel M, Marzari R, et al. Anti transglutaminase antibodies cause ataxia in mice. PloS One. 2010; 5: e9698. doi:10.1371/journal.pone.0009698

28. Bushara KO. Neurologic presentation of celiac disease. Gastroenterology. 2005; 128: S92-S97. doi:10.1053/j.gastro.2005.02.018

29. Anheim M, Degos B, Echaniz-Laguna A, Fleury M, Grucker M, Tranchant C. Ataxia associated with gluten sensitivity, myth or reality? Rev Neurol. 2006; 162: 214-221. doi:10.1016/s00353787(06)75002-0

30. Mitoma H, Adhikari K, Aeschlimann D, Chattopadhyay P, Hadjivassiliou M, Hampe CS, et al. Consensus paper: Neuroimmune mechanisms of cerebellar ataxias. Cerebellum. 2016; 15: 213-232. doi:10.1007/s12311-015-0664-x

31. Cakir D, Tosun A, Polat ME, Celebisoy NE, Gokben SA, Aydogdu S, et al. Subclinical neurological abnormalities in children with celiac disease receiving a gluten-free diet. J Pediatr Gastroenterol Nutr. 2007; 45: 366-369. doi:10.1097/MPG.0b013e31806907e8

32. Rosenberg NR, Vermeulen M. Should coeliac disease be considered in the work up of patients with chronic peripheral neuropathy? J Neurol Neurosurg Psychiatry. 2005; 76: 1415-1419. doi:10.1136/jnnp.2004.048413

33. Cicarelli G, Della Rocca G, Amboni M, Ciacci C, Mazzacca G, Filla A, et al. Clinical and neurological abnormalities in adult celiac disease. Neurol Sci. 2003; 24: 311-317. doi:10.1007/s10072-003-0181-4

34. Wang JL, Yang X, Xia K, Hu ZM, Weng L, Jin X, et al. TGM6 identified as a novel causative gene of spinocerebellar ataxias using exome sequencing. Brain. 2010; 133: 3510-3518. doi:10. 1093/brain/awq323

35. Alaedini A, Green PH, Sander HW, Hays AP, Gamboa ET, Fasano A, et al. Ganglioside reactive antibodies in the neuropathy associated with celiac disease. J Neuroimmunol. 2002; 127: 145148. doi:10.1016/s0165-5728(02)00102-9

36. Polizzi A, Finocchiaro M, Parano E, Pavone P, Musumeci S, Polizzi A. Recurrent peripheral neuropathy in a girl with celiac disease. J Neurol Neurosurg Psychiatry. 2000; 68: 104-105. doi:10.1136/jnnp.68.1.104

37. Bermejo AM. Enfermedad celíaca. Alteraciones neurológicas y psiquiátricas. Bol Pediatr. 2009; 49: 146-156.

38. Lionetti E, Francavilla R, Maiuri L, Ruggieri M, Spina M, Pavone $P$, et al. Headache in pediatric patients with celiac disease and its prevalence as a diagnostic clue. J Pediatr Gastroenterol Nutr. 2009; 49: 202-207. doi:10.1097/MPG.0b013e31818f6389

39. Hadjivassiliou M, Grünewald RA, Lawden M, Davies-Jones GA, Powell T, Smith CM. Headache and CNS white matter abnormalities associated with gluten sensitivity. Neurology. 2001; 56: 385-388. doi:10.1212/wnl.56.3.385

40. Martínez-Bermejo A, Polanco I, Arcas J, López-Martín V, Roche-Herrero MC, Tendero A, et al. Prevalencia de cefalea en una población de enfermos celíacos. Rev Neurol. 2001; 32: 301-309.

41. Hervás Zúñiga A, Balmaña N, Salgado M. Los trastornos del espectro autista (TEA). Pediatría Integral. 2017; XXI: 92-108. 
42. Pavone L, Fiumara A, Bottaro G, Mazzone D, Coleman M. Autism and celiac disease: failure to validate the hypothesis that a link might exist. Biol Psychiatry. 1997; 42: 72-75. doi:10.1016/S0006-3223(97)00267-9

43. Ludvigsson JF, Reichenberg A, Hultman CM, Murray JA. A nationwide study of the association between celiac disease and the risk of autistic spectrum disorders. JAMA Psychiatry. 2013; 70: 1224-1230. doi:10.1001/jamapsychiatry.2013.2048

44. Lau NM, Green PH, Taylor AK, Hellberg D, Ajamian M, Tan CZ, et al. Markers of celiac disease and gluten sensitivity in children with autism. PloS One. 2013; 8: e66155. doi:10.1371/journal.pone.0066155

45. De Lau LML, De Vries JM, Van der Woude CJ, Kuipers EJ, Siepman DA, Sillevis Smitt PA, et al. Acute CNS white matter lesions in patients with inflammatory bowel disease. Inflamm Bowel Dis. 2009; 15: 576-580. doi:10.1002/ibd.20792

46. Martínez-Bermejo A, Polanco I. Alteraciones neuropsicológicas en la enfermedad celíaca. Rev Neurol. 2002; 34: S24-S33.

47. Butwicka A, Lichtenstein P, Frisén L, Almqvist $C$, Larsson $H$, Ludvigsson JF. Celiac disease is associated with childhood psychiatric disorders: A population-based study. J Pediatr. 2017; 184: 87-93. doi:10.1016/j.jpeds.2017.01.043

48. Slim M, Rico-Villademoros F, Calandre EP. Psychiatric comorbidity in children and adults with gluten-related disorders: A narrative review. Nutrients. 2018; 10: 875. doi:10.3390/nu10070875

49. Pynnönen PA, Isometsä ET, Aronen ET, Verkasalo MA, Savilahti E, Aalberg VA. Mental disorders in adolescents with celiac disease. Psychosomatics. 2004; 45: 325-335. doi:10.1176/appi.psy.45.4.325

50. Ludvigsson JF, Reutfors J, Ösby U, Ekbom A, Montgomery SM. Coeliac disease and risk of mood disorders--a general population-based cohort study. J Affect Disord. 2007; 99: 117-126. doi:10.1016/j.jad.2006.08.032

51. Siniscalchi M, lovino P, Tortora R, Forestiero S, Somma A, Capuano L, et al. Fatigue in adult coeliac disease. Aliment Pharmacol Ther. 2005; 22: 489-494. doi:10.1111/j.13652036.2005.02619.x

52. Husby S, Koletzko S, Korponay-Szabó I, Kurppa K, Mearin ML, Ribes-Koninckx C, et al. European society paediatric gastroenterology, hepatology and nutrition guidelines for diagnosing coeliac disease 2020. J Pediatr Gastroenterol Nutr. 2020; 70: 141-156. doi:10.1097/MPG.0000000000002497

53. Bürk K, Melms A, Schulz JB, Dichgans J. Effectiveness of intravenous immunoglobin therapy in cerebellar ataxia associated with gluten sensitivity. Ann Neurol. 2001; 50: 827-828. doi:10.1002/ana.1281 


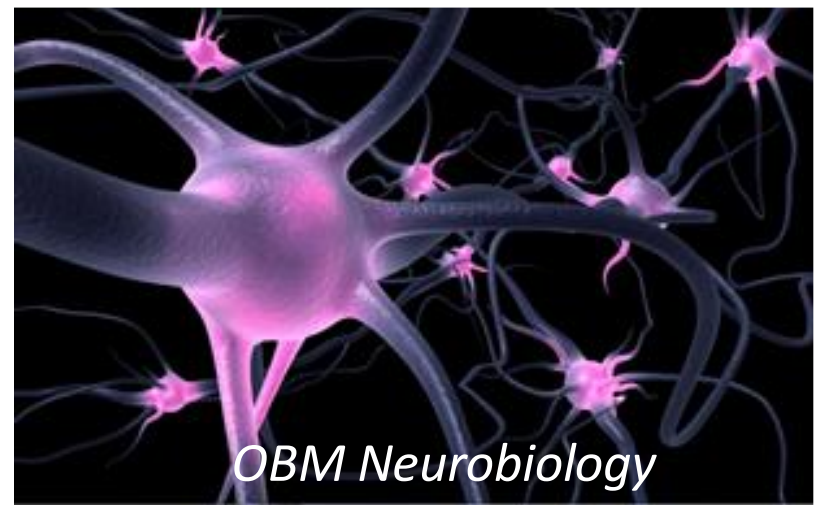

Enjoy OBM Neurobiology by:

1. Submitting a manuscript

2. Joining volunteer reviewer bank

3. Joining Editorial Board

4. Guest editing a special issue

For more details, please visit:

http://www.lidsen.com/journals/neurobiology 